\title{
Reversible postoperative blindness caused by bilateral status epilepticus amauroticus following thoracolumbar deformity correction: case report
}

\author{
Tarik F. Ibrahim, MD, ${ }^{1}$ Rochelle T. Sweis, DO, ${ }^{2}$ and Russ P. Nockels, MD ${ }^{1}$ \\ Departments of ${ }^{1}$ Neurosurgery and ${ }^{2}$ Neurology, Loyola University Medical Center, Maywood, Illinois
}

Postoperative vision loss (POVL) is a devastating complication and has been reported after complex spine procedures. Anterior ischemic optic neuropathy and posterior optic neuropathy are the 2 most common causes of POVL. Bilateral occipital lobe seizures causing complete blindness are rare and have not been reported as a cause of POVL after spine surgery with the patient prone. The authors report the case of a 67-year-old man without a history of seizures who underwent a staged thoracolumbar deformity correction and developed POVL 6 hours after surgery. Imaging, laboratory, and ophthalmological examination results were nonrevealing. Routine electroencephalography study results were negative, but continuous electroencephalography captured bilateral occipital lobe seizures. The patient developed nonconvulsive status epilepticus despite initial treatment with benzodiazepines and loading doses of levetiracetam and lacosamide. He was therefore intubated for status epilepticus amauroticus and received a midazolam infusion. After electrographic seizure cessation for 48 hours, the patient was weaned off midazolam. The patient was maintained on levetiracetam and lacosamide without seizure recurrence and returned to his preoperative visual baseline status.

https://thejns.org/doi/abs/10.3171/2016.12.SPINE16540

KEY WORDS sagittal imbalance; postoperative; complication; blindness; seizure

$\mathrm{P}$ OSTOPERATIVE vision loss (POVL) is a rare but devastating complication after spine surgery. The phenomenon is most commonly related to vascular insults to the optic nerve or occipital cortex following prolonged spinal procedures in the prone position. Arterial ischemia may damage the optic nerve, resulting in either anterior ischemic optic neuropathy (AION) or posterior ischemic optic neuropathy (PION). Occlusion of the central retinal artery may also cause retinal ischemia and postoperative blindness. Less common but previously reported causes of POVL have included cortical blindness due to posterior cerebral artery occlusion or posterior reversible encephalopathy syndrome (PRES).

Occipital lobe seizures resulting in complete blindness are an entity rarely reported in the literature. $3,7,10,12 \mathrm{Re}$ ferred to as status epilepticus amauroticus (SEA), persis- tent occipital lobe seizures most often present with visual hallucinations. However, up to one-third of patients with SEA can develop ictal blindness. ${ }^{1}$ We report a case of a staged thoracolumbar deformity correction complicated by complete cortical blindness secondary to SEA. To our knowledge, this is the first reported case of SEA causing POVL.

\section{Case Report}

History

A 67-year-old man who had not undergone previous spine surgery presented to the clinic complaining of severe low-back pain and bilateral L-3 radiculopathies. His pertinent medical history included hypertension and right nephrectomy following a gunshot wound sustained in

ABBREVIATIONS AION = anterior ischemic optic neuropathy; $\mathrm{EBL}=$ estimated blood loss; $\mathrm{EEG}$ = electroencephalography; PION = posterior ischemic optic neuropathy; $\mathrm{POVL}=$ postoperative vision loss; PRES = posterior reversible encephalopathy syndrome; SEA = status epilepticus amauroticus.

SUBMITTED May 8, 2016. ACCEPTED December 2, 2016.

INCLUDE WHEN CITING Published online April 21, 2017; DOI: 10.3171/2016.12.SPINE16540. 

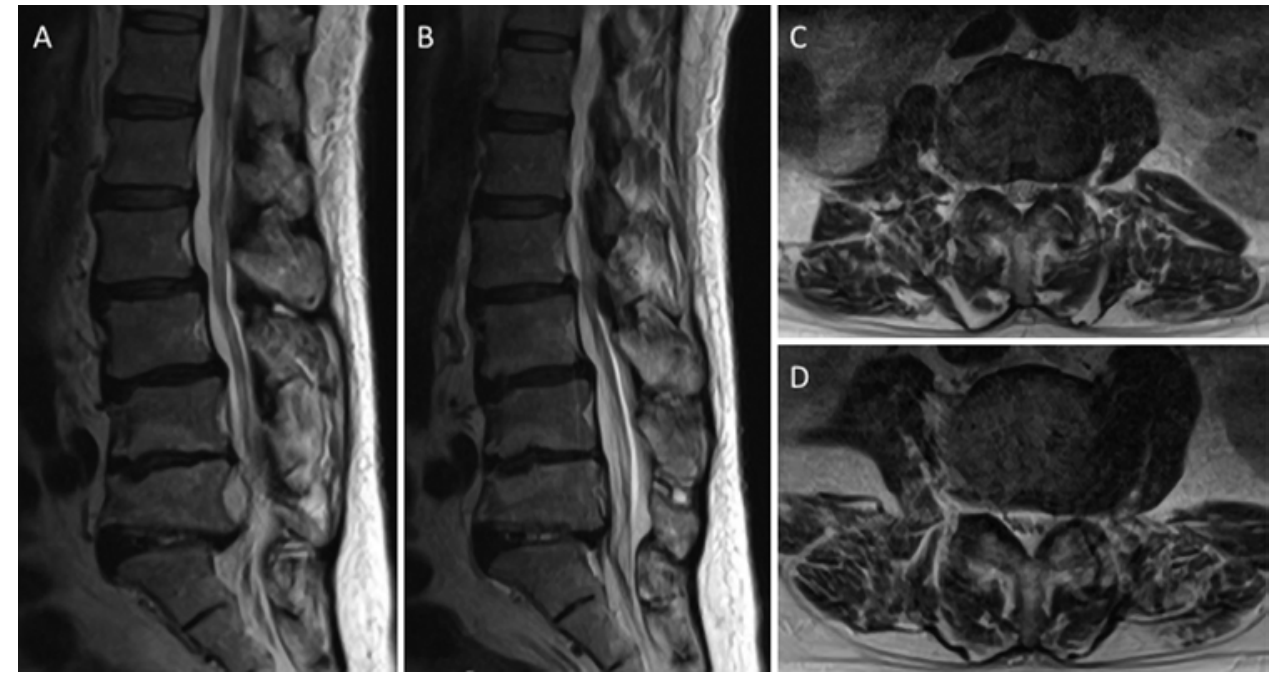

FIG. 1. Preoperative sagittal (A and B) and axial (C [at L2-3] and D [at L3-4]) T2-weighted MR images of the lumbar spine, revealing lumbar and lateral recess stenoses.

military combat. MRI of the lumbar spine revealed diffuse central canal and midlumbar lateral recess stenoses (Fig. 1). Upright long-cassette radiographs revealed severe sagittal imbalance, flattening of normal thoracic kyphosis, and lumbar lordosis-pelvic incidence mismatch (Fig. 2). A lumbar decompression with T3-ilium deformity correction was recommended to alleviate the patient's symptoms. This was planned in a staged fashion with 1 day of rest between the 2 stages.

\section{Operation}

During the first operation, we performed T-12 through S-1 laminectomies, medial facetectomies, and posterior segmental instrumentation of T-12 to the ilium with
Smith-Petersen osteotomies of L-2, L-3, and L-4. The estimated blood loss (EBL) was $3000 \mathrm{ml}$. The patient awoke at his neurological baseline; however, the second stage was delayed by 6 days due to development of reversible acute kidney injury and ileus, both of which were treated and resolved before the second procedure. The acute kidney injury was secondary to the presence of only 1 kidney and mild intraoperative hypotension that responded to colloid, packed red blood cells, and intravenous phenylephrine. The second stage was again uncomplicated, during which the planned T3-ilium instrumented correction was completed. The EBL during the second stage was 1800 $\mathrm{ml}$. During the procedure, the patient received $5200 \mathrm{ml}$ crystalloid, $750 \mathrm{ml} \mathrm{5 \%}$ albumin, and $5 \mathrm{U}$ packed red blood
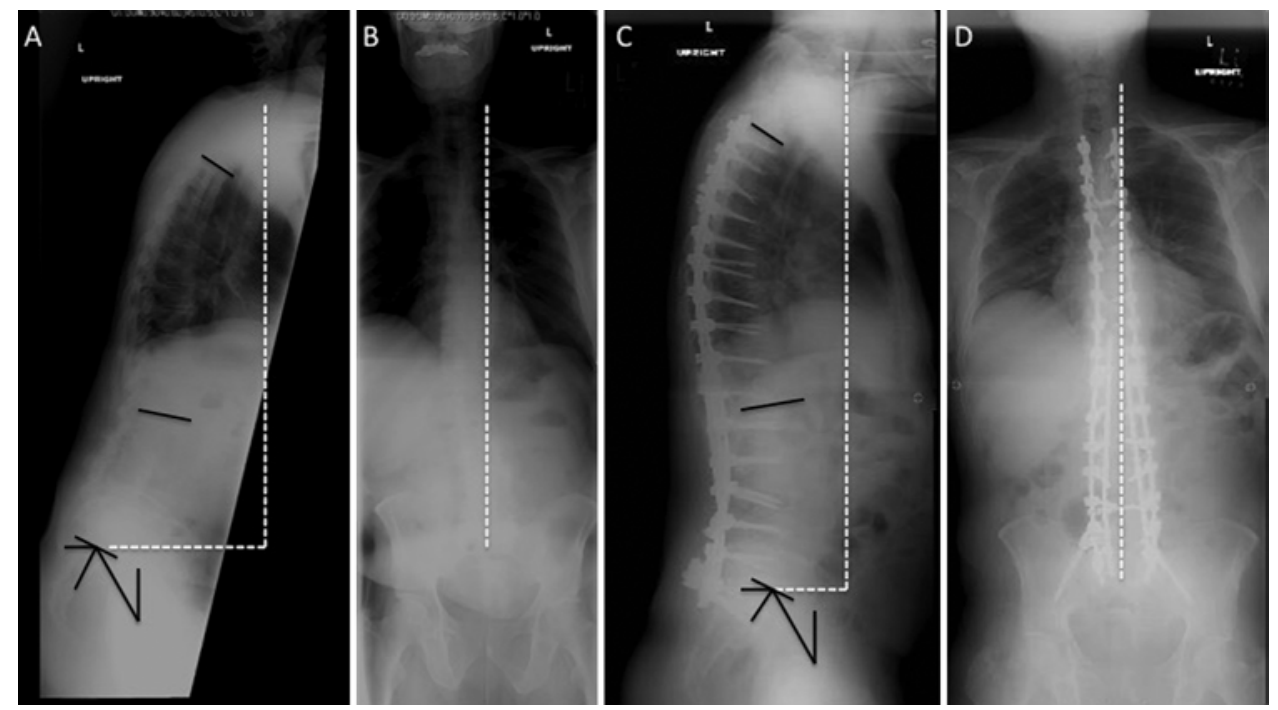

FIG. 2. Preoperative lateral (A) and anteroposterior (B) standing long-cassette radiographs revealing sagittal imbalance with flat-back deformity (pelvic incidence, $50^{\circ}$; pelvic tilt, $29^{\circ}$; lumbar lordosis, $8^{\circ}$; sagittal vertical axis, positive $17 \mathrm{~cm}$; and thoracic kyphosis, $18^{\circ}$ ). Postoperative lateral (C) and anteroposterior (D) studies showing improvement in sagittal balance with increased lumbar lordosis and thoracic kyphosis (pelvic incidence, $50^{\circ}$; pelvic tilt, $29^{\circ}$; lumbar lordosis, $25^{\circ}$; sagittal vertical axis, positive $5 \mathrm{~cm}$; and thoracic kyphosis, $\left.36^{\circ}\right)$. The black lines indicate the preoperative and postoperative angles, and the dotted lines depict the sagittal and coronal balance. 
cells. The patient was maintained normotensive throughout the procedure without the use of pressor support, and hourly hemoglobin values were never less than $9.9 \mathrm{~g} / \mathrm{dl}$.

\section{Postoperative Course}

The patient awoke from the second stage of the procedure at his neurological baseline with intact vision. Six hours later, he complained of sudden-onset visual loss. On examination, he was found to have bilateral blindness with absent perception of light or hand motion. He was also noted to be disoriented to year and location with full body rigidity and a left facial droop. Intravenous levetiracetam was empirically administered, and the patient continued on maintenance therapy. Findings of a noncontrast CT scan of the head was negative for acute pathology. This was followed by MRI, MR angiography, and MR venography of the brain, the findings of which were all unremarkable (Fig. 3). Results of a comprehensive altered mental status laboratory workup revealed no abnormalities. Carotid duplex and echocardiography findings were both normal. Routine electroencephalography (EEG) revealed slowing of the posterior background, but it did not reveal epileptiform discharges or seizure activity. Ophthalmology examination revealed a left pupil fixed at $3 \mathrm{~mm}$ and a right pupil fixed at $1 \mathrm{~mm}$. The bilateral pupils briefly dilated when light was withdrawn and then slowly returned to their previous size without a light stimulus. Switching from dark to light exposure did not elicit pupillary dilation or constriction. A dilated funduscopic examination revealed intact bilateral anterior optic nerves and no abnormal retinal findings. Medication side effects were entertained to explain these findings, but no clear ophthalmological etiology could explain the pupillary findings.

As the ophthalmological examination results were inconclusive and the patient continued to be intermittently confused, continuous video-EEG was started 2 days after the second stage of surgery. This revealed bilateral occipital lobe seizures (Fig. 4) with an associated semiology of confusion and blindness only. Nystagmus and eye deviation were absent. The seizures temporarily stopped after the patient received 3 doses of intravenous lorazepam 2 $\mathrm{mg}$, intravenous levetiracetam $1000 \mathrm{mg}$, and lacosamide $400 \mathrm{mg}$. However, the patient remained blind. Bioccipital electrographic status epilepticus recurred 1 hour later. The patient was therefore intubated, and a continuous intravenous infusion of midazolam was initiated with a loading dose of $0.2 \mathrm{mg} / \mathrm{kg}$ and continued at $0.1 \mathrm{mg} / \mathrm{kg} / \mathrm{hr}$ with resolution of occipital status epilepticus. The midazolam infusion was slowly titrated off over 3 days with the patient maintained on a regimen of levetiracetam and lacosamide. No recurrent seizures were found on EEG. The patient was subsequently extubated and was found to have regained his baseline vision. The ophthalmology service found his visual acuity to be $20 / 40$ in the right eye and $20 / 30$ in the left eye without any field cuts. The patient is presently less than 1 year out of surgery; his construct is stable and his vision remains intact.

\section{Discussion}

POVL is a devastating complication after any surgery.
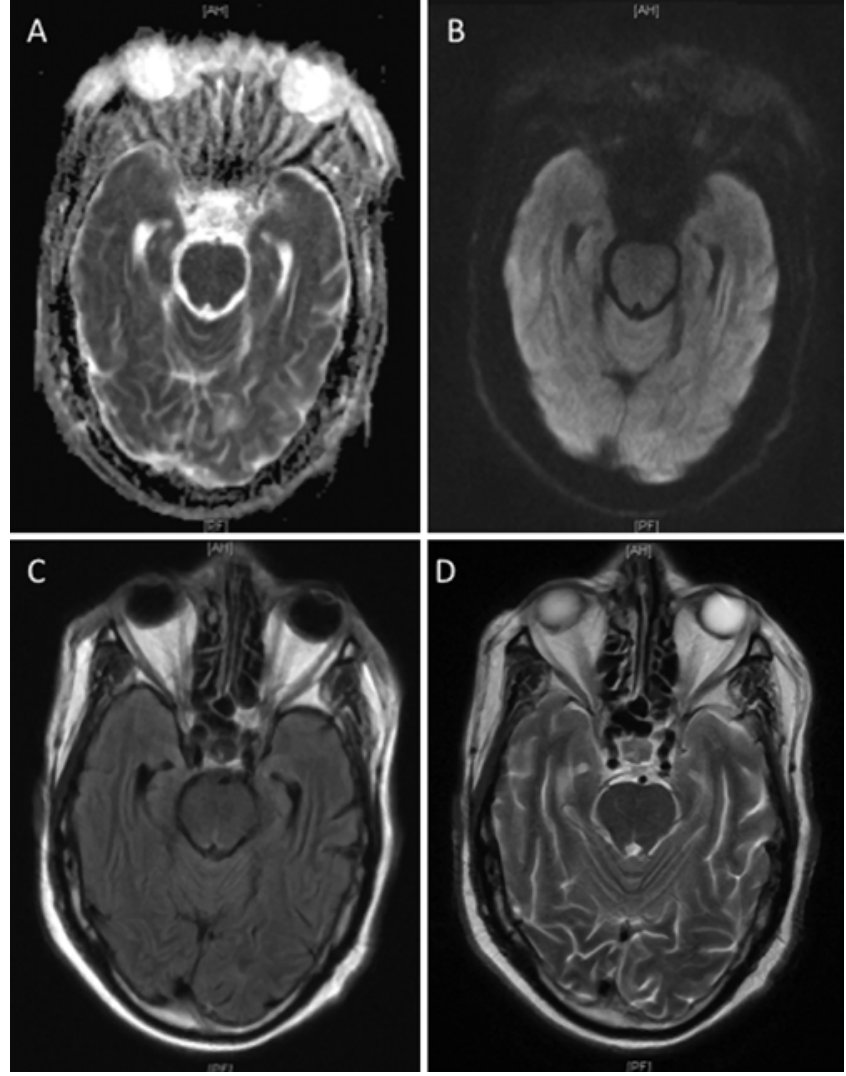

FIG. 3. Apparent diffusion coefficient (A), diffusion-weighted (B), FLAIR (C), and T2-weighted (D) axial MR imaging sequences of the brain do not reveal restricted diffusion, mass lesions, or any other possible explanation for the patient's complete blindness.

It is most frequently reported after spine and cardiac surgery with estimates of $0.2 \%$ and $4.5 \%$, respectively. ${ }^{11,13}$ Etiologies for POVL after spine surgery include AION, PION, central retinal artery occlusion, PRES, and cortical blindness due to posterior cerebral artery territory ischemia. The American Society of Anesthesiologists POVL Registry reports that AION and PION account for $89 \%$ of cases of POVL after spine surgery. ${ }^{8}$ The Perioperative Visual Loss Study Group found that independent risk factors of AION and PION were male sex, obesity, use of the Wilson frame, prolonged duration of surgery, significant blood loss, and a low colloid-to-crystalloid administration ratio. ${ }^{9}$ Pituitary apoplexy following spine surgery has been reported to cause postoperative bitemporal hemianopia but not complete blindness. ${ }^{2}$ To our knowledge, this is the first reported case of occipital lobe status epilepticus causing complete blindness after spine surgery.

Reported cases of SEA are also rare. Barry et al. ${ }^{3}$ reported a case series of 5 patients with SEA, 3 of whom were completely blind. The remaining patients experienced a homonymous hemianopia. Varied etiologies of the seizures in the 3 cases of complete blindness were implicated (1 occipital vascular lesion, 1 occipital neoplasm, and 1 was regarded as idiopathic).

Shaw et al. ${ }^{12}$ described 3 patients with seizure histories (only 2 with epilepsy) who developed SEA that manifested as homonymous hemianopia. None of their patients had 


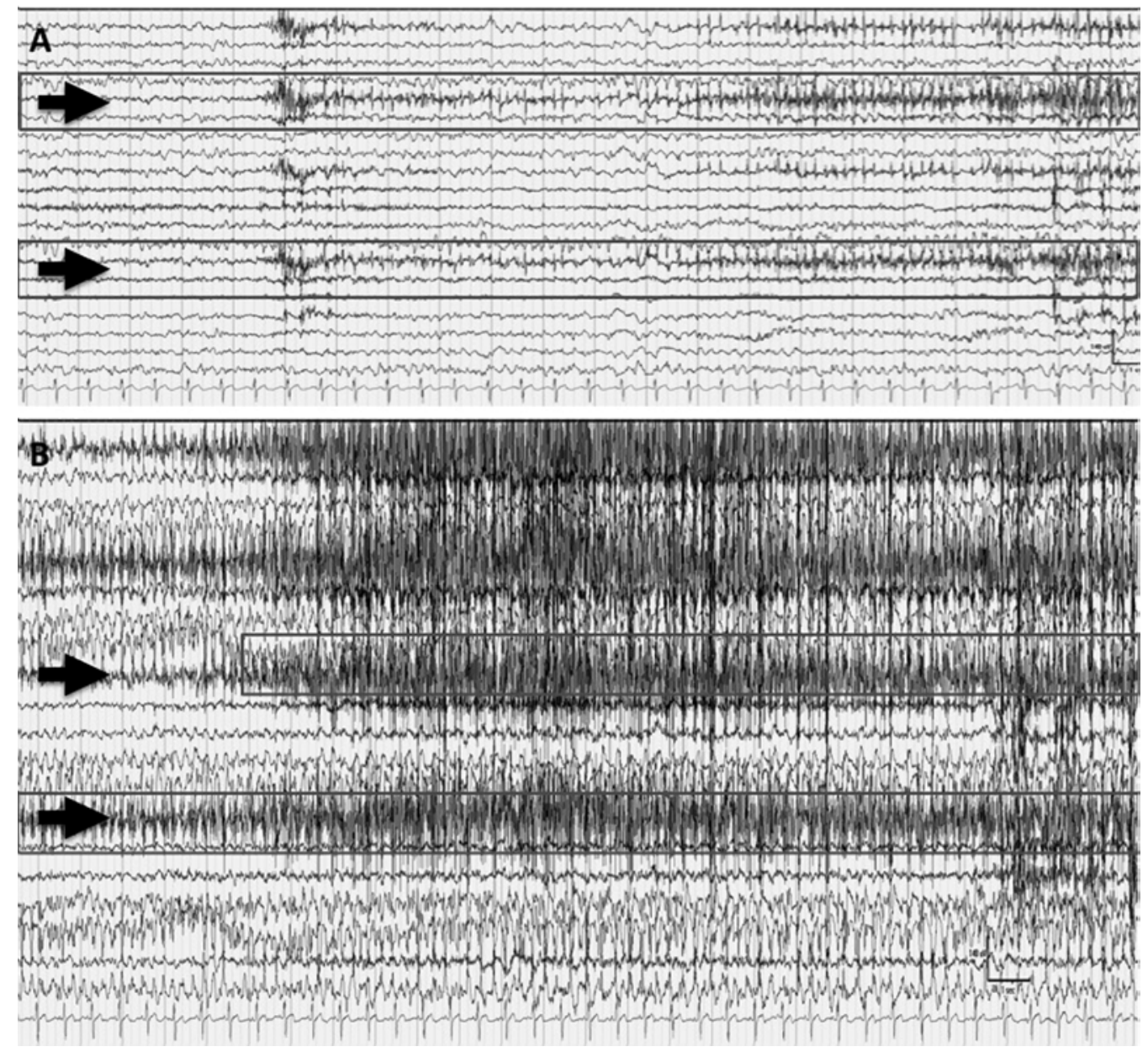

FIG. 4. The initial EEG study reveals partial seizures consisting of frequent occipital spikes, isolated or organized in clusters, arising from the right and left occipital regions ( $\mathbf{A}$, arrows). The partial bioccipital seizures eventually become generalized and persistent (B, arrows).

undergone surgery. All 3 patients returned to their visual baseline over several weeks with antiepileptic drugs. Compared with these 3 patients, our patient did not have a history of epilepsy, and SEA was not observed on MRI. Other authors have reported SEA to occur idiopathically, which is the scenario in this case.

\section{Occipital Lobe Seizures}

Occipital lobe epilepsy accounts for 5\%-10\% of all epilepsies and may present at any age, except for idiopathic occipital lobe epilepsy, which begins in childhood. Ictal symptoms are visual and/or oculomotor. Tonic deviation of the eyes remains the most common oculomotor manifestation, often combined with ipsilateral turning of the head. Nystagmus and repetitive eyelid closure or eyelid fluttering are also commonly observed. ${ }^{1}$ Visual symptoms include visual hallucinations and illusions, sensory hallucinations of ocular movements, palinopsia, and both positive and negative phenomena. ${ }^{1}$

Positive features include flashes and scintillations. Although rare, negative manifestations such as cortical blindness can occur. Seizures may be unilateral or bilateral and therefore present with homonymous hemianopia or complete ictal blindness, respectively. Occipital seizures may last seconds to minutes, with SEA being much less common. Visual loss in seizing patients can persist from days to weeks without cognitive dysfunction and may be associated with scintillations, diplopia, and simple motor seizures. Vision typically returns to baseline with aggressive anticonvulsant treatment. Although not clearly defined in cases of SEA, permanent neurological deficit is known to be associated with untreated status epilepticus elsewhere in the brain due to cortical laminar necrosis of the affected gyri. ${ }^{6}$

\section{Anesthesia, SEA, and POVL}

The effect of anesthesia in patients with intractable partial epilepsy undergoing surgery has previously been evaluated, but the incidence of clinical seizure activity in individuals without epilepsy in response to general anesthetic agents remains unknown..$^{14}$ Because certain anesthetic medications carry a proconvulsant effect, it is necessary to understand the consequences of their use in patients with epilepsy in whom general anesthesia is induced. Epileptiform activity has been demonstrated on EEG in patients receiving anesthetic agents, but clinical or electro- 
graphic seizures are rare. Anesthetic medications which may lower seizure threshold include the inhaled volatile anesthetics (enflurane, sevoflurane), local anesthetics (lidocaine, bupivacaine), opiates (fentanyl, sufentanil, alfentanil, meperidine), and even sedative/hypnotic anesthetics including etomidate, ketamine, and methohexital. ${ }^{4}$

The use of motor evoked potentials carries with it the theoretical risk of inducing postoperative seizures in patients without a seizure history. This, however, is an extremely rare phenomenon and would more likely involve the corticospinal tracts and not the occipital cortex. ${ }^{5}$

\section{SEA Management}

SEA can initially be managed with benzodiazepines, followed by the intravenous administration of levetiracetam, valproic acid, or phenytoin. For status epilepticus, a continuous infusion of either midazolam or propofol with intubation and hemodynamic support using intravenous fluids and vasopressors should be initiated. These are the measures that were undertaken in our patient. Pentobarbital, ketamine, and high volatile anesthetics (isoflurane, desflurane, halothane) can be used for refractory and super-refractory status epilepticus. ${ }^{1}$

\section{Conclusions}

POVL is a devastating and rare complication associated with cardiac and spine surgery. The most common etiologies are AION and PION. Occipital lobe seizures and SEA presenting as complete blindness may represent an etiology for POVL and should be considered in patients regardless of seizure history. Clinical suspicion is raised if seizure semiology is accompanied by altered mental status, eye deviation, or body shaking and rigidity. Continuous EEG is required for diagnosis, as routine EEG may not capture nonconvulsive seizures and SEA. Treatment with antiepileptic drugs should be initiated for occipital lobe seizures and may require intubation for continuous intravenous infusions (midazolam, pentobarbital, ketamine, volatile anesthetics) if SEA develops.

This report describes SEA as a novel cause of POVL that requires a heightened degree of clinical suspicion. If left untreated, SEA may result in permanent visual loss. However, as demonstrated in this case report, early recognition and aggressive antiseizure treatment can result in an excellent outcome.

\section{References}

1. Adcock JE, Panayiotopoulos CP: Occipital lobe seizures and epilepsies. J Clin Neurophysiol 29:397-407, 2012

2. Akakıı A, Yılmaz B, Ekşi MS, Kılıç T: A case of pituitary apoplexy following posterior lumbar fusion surgery. J Neurosurg Spine 23:598-601, 2015
3. Barry E, Sussman NM, Bosley TM, Harner RN: Ictal blindness and status epilepticus amauroticus. Epilepsia 26:577584,1985

4. Benish SM, Cascino GD, Warner ME, Worrell GA, Wass CT: Effect of general anesthesia in patients with epilepsy: a population-based study. Epilepsy Behav 17:87-89, 2010

5. Davis SF, Altstadt T, Flores R, Kaye A, Oremus G: Report of seizure following intraoperative monitoring of transcranial motor evoked potentials. Ochsner J 13:558-560, 2013

6. Donaire A, Carreno M, Gómez B, Fossas P, Bargalló N, Agudo R, et al: Cortical laminar necrosis related to prolonged focal status epilepticus. J Neurol Neurosurg Psychiatry 77:104-106, 2006

7. Ghosh P, Motamedi G, Osborne B, Mora CA: Reversible blindness: simple partial seizures presenting as ictal and postictal hemianopsia. J Neuroophthalmol 30:272-275, 2010

8. Lee LA, Roth S, Posner KL, Cheney FW, Caplan RA, Newman NJ, et al: The American Society of Anesthesiologists Postoperative Visual Loss Registry: analysis of 93 spine surgery cases with postoperative visual loss. Anesthesiology 105:652-659, 867-658, 2006

9. Postoperative Visual Loss Study Group: Risk factors associated with ischemic optic neuropathy after spinal fusion surgery. Anesthesiology 116:15-24, 2012

10. Sawchuk KS, Churchill S, Feldman E, Drury I: Status epilepticus amauroticus. Neurology 49:1467-1469, 1997

11. Shaw PJ, Bates D, Cartlidge NE, Heaviside D, French JM, Julian DG, et al: Neuro-ophthalmological complications of coronary artery bypass graft surgery. Acta Neurol Scand 76:1-7, 1987

12. Shaw S, Kim P, Millett D: Status epilepticus amauroticus revisited: ictal and peri-ictal homonymous hemianopsia. Arch Neurol 69:1504-1507, 2012

13. Stevens WR, Glazer PA, Kelley SD, Lietman TM, Bradford DS: Ophthalmic complications after spinal surgery. Spine (Phila Pa 1976) 22:1319-1324, 1997

14. Wass CT, Grady RE, Fessler AJ, Cascino GD, Lozada L, Bechtle PS, et al: The effects of remifentanil on epileptiform discharges during intraoperative electrocorticography in patients undergoing epilepsy surgery. Epilepsia 42:1340-1344, 2001

\section{Disclosures}

Dr. Nockels reports that he is a consultant for Medtronic.

\section{Author Contributions}

Conception and design: all authors. Acquisition of data: all authors. Analysis and interpretation of data: all authors. Drafting the article: all authors. Critically revising the article: all authors. Reviewed submitted version of manuscript: all authors.

\section{Correspondence}

Russ P. Nockels, Department of Neurosurgery, Loyola University Medical Center, 2160 South First Ave., Bldg. 105, Rm. 1900, Maywood, IL 60153. email: rpnockels@mac.com. 